\title{
A robust method to identify meteor showers new parent bodies from the SonotaCo and EDMOND meteoroid orbit databases ${ }^{\star}$
}

\author{
M. Guennoun ${ }^{1,2}$, J. Vaubaillon ${ }^{1}$, D. Čapek ${ }^{4}$, P. Koten ${ }^{4}$, and Z. Benkhaldoun ${ }^{2,3}$ \\ ${ }^{1}$ Institut de Mécanique celeste et calcul des Éphémérides, Observatoire de Paris, PSL, France \\ ${ }^{2}$ Laboratory of High Energy Physics and Astrophysics, Physics Department, Faculty of Science Semlalia, Cadi Ayyad University, \\ PO Box 2390, Marrakesh 40000, Morocco \\ e-mail: meryem.guennoun@ced.uca.ac.ma \\ 3 Oukaimeden Observatory, Cadi Ayyad University, PO Box 2390, Marrakesh 40000, Morocco \\ ${ }^{4}$ Astronomical Institute of Academy of Sciences, Fričova 298, 25165 Ondčejov, Czech Republic
}

Received 7 November 2018 / Accepted 7 December 2018

\begin{abstract}
Context. Several new meteor showers are added to the International Astronomical Union (IAU) list of meteor showers every year. Given the multiplication of video meteor measurements new parent bodies are to be found in addition to new showers. Such an endeavor is usually performed by comparing orbital elements, using a high threshold single-linking $D_{\text {sh-criterion. However, questions }}$ remain about the accuracy of the method and the veracity of the newly associated parent bodies. Aims. Our goal is to find the presence of new parent bodies in a statistical meaningful way.

Methods. A search for parent bodies was performed among SonotaCo and EDMOND databases. The association of asteroids with meteors was based on different methods, discussed and compared below. In addition, a thorough statistical test was performed in order to investigate the possible random associations.

Results. A list of potential new parent bodies associated with at least 50 meteors is found. A statistical test was used to show whether the group of meteor orbits and the asteroid is a random coincidence or not. Out of 54 potential new parent bodies, only three new parent bodies are not excluded by the statistical test: $2014 B N_{14}, 2015 T X_{24}$ and $2015 Q T_{3}$, with a probability of chance occurrence of 37,10 and $13 \%$, respectively. This shows the need for rigorous method when searching for the existence of meteor showers and parent bodies.

Conclusions. Ideally, such a test (or even better, tests) should be conducted in order to confirm (or not) the current IAU list of meteor showers. Similarly, all meteor orbit data in our databases should ideally be revisited using the latest methods providing a better estimate of the real uncertainty and accuracy of the derived orbits.
\end{abstract}

Key words. methods: data analysis - methods: numerical - meteorites, meteors, meteoroids - minor planets, asteroids: general

\section{Introduction}

The International Astronomical Union (IAU) keeps track of all known meteor showers and their parent bodies, including those in need of confirmation. New showers are regularly added from ongoing meteoroid orbit surveys (Jenniskens et al. 2009; Jopek \& Kaňuchová 2017).

In early 2018, the IAU Meteor Data Center lists 957 meteor showers, including 112 established and others considered in the working list ${ }^{1}$. Recently, Kornoš et al. (2014); Rudawska et al. (2015a,b); Jenniskens \& Nénon (2016); Rudawska \& Jenniskens (2014); Jenniskens et al. (2016) have identified several new meteor showers that were added to the IAU list of Meteor Showers using a high threshold single-linking $D_{\text {sh }}$-criterion (Southworth \& Hawkins 1963).

Here we investigate the possible parent bodies of these new showers. Usually, such a search is performed by comparing the orbits of known asteroids and comets to the mean orbit of a given

\footnotetext{
$\star$ Tables of all the associations are only available at the CDS via anonymous ftp to cdsarc.u-strasbg.fr (130.79.128.5) or via http://cdsarc.u-strasbg.fr/viz-bin/qcat?J/A+A/622/A84 1 http://pallas .astro.amu.edu.pl/ jopek/MDC2007/index. php, accessed in Feb 2018.
}

shower, by using for example one of the $D_{\mathrm{sh}}$-criterion defined by several authors (e.g. Jopek 1993; Rudawska \& Jenniskens 2014; Rudawska et al. 2015b). However, in recent work, doubts have been raised regarding the veracity of such a method, as a dependency on the sample was unveiled (Jopek \& Bronikowska 2017). Indeed, as already shown in the case of the Pribram and Neuschweinstein meteorites, orbit similarity does not necessarily imply a common origin and thorough statistical analysis is needed to draw robust conclusions (regardless of the composition of the meteorites Pauls \& Gladman 2005; Koten et al. 2014). Thanks to the multiplication of video meteor networks, the number of publicly available meteor orbits is growing quickly, and so it is not surprising to find many meteors with orbits similar to known asteroids or comets. In addition, the uncertainties of meteoroid orbital elements are much higher than expected before, as showed by Egal et al. (2017); Vida et al. (2018). Nevertheless, the need for parenthood for meteor shower is crucial in order to better perform typical dynamic works such as the exploration of the age of a meteoroid stream or the forecasting of future meteor showers (Abedin et al. 2017; Vaubaillon 2017). The question thus remains whether or not a given association between a meteor (or set of meteors) and parent body is relevant. 
In this paper, we present a new method, based on statistical analysis, to compare the parent bodies and meteoroids orbits. The method is presented in Sect. 2 and the results in Sect. 3. A discussion regarding the veracity of the developed method and the comparison to other ones follows in Sects. 3 and 4.

\section{Method}

\subsection{Data}

We use the video meteor orbits of SonotaCo and Edmond Database to find associations with a list of 15129 asteroids taken from JPL Small body Database ${ }^{2}$ that provides us with tables of orbital and/or physical parameters for all existing asteroids and comets. The SonotaCo video meteor network operates about 100 cameras at 25 sites. It has been in operation since 2007 , with first results published in 2009 (SonotaCo 2009; Jenniskens et al. 2016). Every year, new orbits are published on the SonotaCo website ${ }^{3}$ by Touru Kanamori, Tokyo, Japan. From 2007 to 2017, the SonotaCo database grew to 257104 orbits. The computation of meteor orbits is performed by the UFOOrbit software (SonotaCo 2009).

The European video Meteor Network Database (EDMOND) is a database of video meteor orbits resulting from a cooperation and data sharing between several European national networks as well as the International Meteor Organization Video Meteor Network IMO VMN (Rudawska et al. 2015a). In early 2018, the EDMOND database contains 317831 video meteor orbits, gathered from 2001 to $2016^{4}$. A comparison of both the EDMOND and the SonotaCo catalogue, in terms of orbital parameters, showed an equivalence of the data (Kornoš et al. 2014).

\subsection{Searching for an appropriate method}

Most modern research on the association between parent bodies and meteor showers uses the Dissimilarity Criterion or the D criterion, which was first proposed by Southworth \& Hawkins (1963) and has been modified by others, for example, Drummond (1981); Jopek (1993); Rudawska et al. (2015b). A small $D$-value indicates a high degree of similarity between two orbits. A threshold value of $D_{\mathrm{sh}}<0.25$ is often used (Lindblad 1971a,b; SonotaCo 2009; Andreić et al. 2013; Šegon et al. 2014). A lower threshold value of $D_{\mathrm{sh}}<0.05$ was used by Rudawska \& Jenniskens (2014); Kornoš et al. (2014); and Jenniskens \& Nénon (2016). Rudawska et al. (2012, 2015b) have revised such methods but there is still room for improvements, since even the date of ejection of simulated meteoroid stream cannot be found by backward integrations. The main difficulty remains in the comparison of semi-major axis and eccentricity, which are poorly determined (Egal et al. 2017; Vida et al. 2018). In order to bypass this difficulty, Micheli (2013) compares parent bodies and meteor showers orbits based on the comparison of the velocity and location of the radiant rather than the $D_{\mathrm{sh}}$-criterion.

Presumably, as this method uses better-constrained parameters, we developed a method that compares the apparent radiant and geocentric velocity $\left(\alpha, \delta, V_{g}\right)$ of meteors and known potential parent bodies. The radiant and velocities of NEOs (asteroids and comets) can be computed thanks to several method, all summed up by Neslusan et al. (1998). The program is available on request and was kindly provided to us by the author. This step is performed using six different methods (Neslusan et al. 1998):

\footnotetext{
2 https://ssd.jpl . nasa. gov accessed in Feb 2018.

3 http://sonotaco.com/e_index.html

4 Accessed in Feb 2018.
}

- adjustment of the orbit by variation of the perihelion distance - the q-adjustment (Hasegawa 1990);

- adjustment of the orbit by variation of both the perihelion distance and eccentricity considering a minimum change (Svoren et al. 1993);

- adjustment of the orbit by variation of the argument of perihelion (Steel \& Baggaley 1985);

- adjustment of the orbit by rotation around the line of apsides (Svoren et al. 1993);

- adjustment of the orbit by variation of the argument of perihelion and inclination (Hasegawa 1990);

- parallel shift of the velocity vector (Porter 1952).

Most of the time, we considered the Hasegawa (1990) method.

The next step is to compare both the meteors $\left(\alpha_{m}, \delta_{m}, V_{m}^{g}\right)$ and the parent bodies $\left(\alpha_{\mathrm{NEO}}, \delta_{\mathrm{NEO}}, V_{\mathrm{NEO}}^{g}\right)$. As a first implementation, one might consider several criteria in terms of difference of position of the radiant $\left(\Delta_{c}^{\mathrm{rad}}\right)$ and difference in velocity $\left(\Delta V_{c}^{g}\right)$ are defined. To consider the parenthood to be possible, a meteor and an NEO must satisfy: $\Delta^{\mathrm{rad}}<\Delta_{c}^{\mathrm{rad}}$ and $\Delta V^{g}<\Delta V_{c}^{g}$. This method resulted in association of the 2009 WN25 asteroid and the November i-Draconids (NID) by Micheli et al. (2016).

In our quest for the most appropriate method, the comparison of methods based on orbital elements and methods based on $\left(\alpha, \delta, V^{g}\right)$ was performed. As a first step, the $D_{\text {sh }}$ (Southworth \& Hawkins 1963) criterion is computed, coupled with a constrain on Solar longitude in order to take into account the apsidal precession of the orbit and to focus on young showers only. Trying different threshold values, we considered: $8^{\circ}$ for the difference in solar longitude, and $0.05,0.1$ and 0.2 for $D_{\text {sh }}$. Such a method is referred to as Method 1 in Tables 1 and 2.

As a second step, and inspired by Micheli (2013), we computed the angular distance between the radiants, and the difference in solar longitude and Velocity. We fixed the threshold values for the difference in the position of the radiant and in the solar longitude, respectively, at $8^{\circ}$ (solar longitude). In order to explore and find the best compromise, several values of the thresholds for the difference of position of radiant and the difference of velocity are considered. In a first step, the distance to the radiant is taken as $6^{\circ}$. Then it is refined at $2^{\circ}$. Similarly, the difference in velocity is taken at $0.5,3$ and $5 \mathrm{~km} \mathrm{~s}^{-1}$. This method is referred to as Method 2. Given these choices, six different combinations of the difference in radiant and velocity are possible.

In order to fully explore the influence of the velocity, in addition to the criteria in solar longitude and radiant position, we considered a variable velocity threshold, based on a percentage of the measured meteoroid velocity. It is worth mentioning that the UFOOrbit orbit computation sofwtare provides a so called $\mathrm{dv} 12 \%$ parameter, characterizing the quality of the orbit, and was commented in, for example, Kornoš et al. (2014). In our work, we have considered that an NEO is a potential parent body if $V g-V g * v \%<V_{\mathrm{NEO}}^{g}<V g+V g * v \%$, with the thresholds $v \%$ taken as $v \%=5,10$ or $20 \%$ of the meteoroid velocity. In other words, the NEO geocentric velocity must be comprised within the uncertainty of the meteoroid geocentric velocity for the association to be considered possible. This last method is called Method 3 hereafter.

Last but not least, in order for a shower to be confirmed and a parent body to be possible, a minimum number of $N_{m}$ meteors must satisfy all the criteria for a given method. Trying several values, we retain $N_{m} \geq 10$ or 50 for the parenthood to be further considered. The results of such a preliminary exploration are shown in Table 1 for $N_{m} \geq 10$ and Table 2 for $N_{m} \geq 50$. 
Table 1. Number of asteroids associated with at least ten meteors, for each method.

\begin{tabular}{|c|c|c|c|c|}
\hline \multirow{2}{*}{ Methods } & \multirow{2}{*}{ \# } & \multirow{2}{*}{ Criteria } & \multicolumn{2}{|c|}{ Number of associations } \\
\hline & & & SonotaCo Database & EDMOND Database \\
\hline \multirow{3}{*}{ Method 1} & 1.1 & $D_{\mathrm{sh}}<0.05, \lambda_{\mathrm{o}}<8^{\circ}$ & 6 & 11 \\
\hline & 1.2 & $D_{\mathrm{sh}}<0.1, \lambda_{\mathrm{o}}<8^{\circ}$ & 420 & 638 \\
\hline & 1.3 & $D_{\mathrm{sh}}<0.2, \lambda_{\mathrm{o}}<8^{\circ}$ & 3230 & 3839 \\
\hline \multirow{6}{*}{ Method 2} & 2.1 & $V<0.5 \mathrm{~km} \mathrm{~s}^{-1}$, Dis $<6^{\circ}, \lambda_{\mathrm{o}}<8^{\circ}, \alpha<8^{\circ}, \delta<8^{\circ}$ & 190 & 368 \\
\hline & 2.2 & $V<1 \mathrm{~km} \mathrm{~s}^{-1}$, Dis $<6^{\circ}, \lambda_{\mathrm{o}}<8^{\circ}, \alpha<8^{\circ}, \delta<8^{\circ}$ & 513 & 929 \\
\hline & 2.3 & $V<3 \mathrm{~km} \mathrm{~s}^{-1}$, Dis $<6^{\circ}, \lambda_{\mathrm{o}}<8^{\circ}, \alpha<8^{\circ}, \delta<8^{\circ}$ & 1519 & 2267 \\
\hline & 2.4 & $V<0.5 \mathrm{~km} \mathrm{~s}^{-1}$, Dis $<2^{\circ}, \lambda_{\mathrm{o}}<8^{\circ}, \alpha<8^{\circ}, \delta<8^{\circ}$ & 115 & 231 \\
\hline & 2.5 & $V<1 \mathrm{~km} \mathrm{~s}^{-1}$, Dis $<2^{\circ}, \lambda_{\mathrm{o}}<8^{\circ}, \alpha<8^{\circ}, \delta<8^{\circ}$ & 350 & 709 \\
\hline & 2.6 & $V<3 \mathrm{~km} \mathrm{~s}^{-1}$, Dis $<2^{\circ}, \lambda_{\mathrm{o}}<8^{\circ}, \alpha<8^{\circ}, \delta<8^{\circ}$ & 1256 & 1975 \\
\hline \multirow{3}{*}{ Method 3} & 3.1 & $\mathrm{dv}=5 \%$, Dis $<2^{\circ}, \lambda_{\mathrm{o}}<8^{\circ}, \alpha<8^{\circ}, \delta<8^{\circ}$ & 315 & 555 \\
\hline & 3.2 & $\mathrm{dv}=10 \%$, Dis $<2^{\circ}, \lambda_{\mathrm{o}}<8^{\circ}, \alpha<8^{\circ}, \delta<8^{\circ}$ & 315 & 555 \\
\hline & 3.3 & $\mathrm{dv}=20 \%$, Dis $<2^{\circ}, \lambda_{\mathrm{o}}<8^{\circ}, \alpha<8^{\circ}, \delta<8^{\circ}$ & 1452 & 2150 \\
\hline
\end{tabular}

Table 2. Number of asteroids associated with at least 50 meteors, for each method.

\begin{tabular}{|c|c|c|c|c|}
\hline \multirow{2}{*}{ Methods } & \multirow{2}{*}{ \# } & \multirow{2}{*}{ Criteria } & \multicolumn{2}{|c|}{ Number of associations } \\
\hline & & & SonotaCo Database & EDMOND Database \\
\hline \multirow{3}{*}{ Method 1} & 1.1 & $D_{\mathrm{sh}}<0.05, \lambda_{\mathrm{o}}<8^{\circ}$ & 2 & 3 \\
\hline & 1.2 & $D_{\mathrm{sh}}<0.1, \lambda_{\mathrm{o}}<8^{\circ}$ & 17 & 28 \\
\hline & 1.3 & $D_{\mathrm{sh}}<0.2, \lambda_{\mathrm{o}}<8^{\circ}$ & 850 & 1424 \\
\hline \multirow{6}{*}{ Method 2} & 2.1 & $V<0.5 \mathrm{~km} \mathrm{~s}^{-1}$, Dis $<6^{\circ}, \lambda_{\mathrm{o}}<8^{\circ}, \alpha<8^{\circ}, \delta<8^{\circ}$ & 16 & 25 \\
\hline & 2.2 & $V<1 \mathrm{~km} \mathrm{~s}^{-1}$, Dis $<6^{\circ}, \lambda_{\mathrm{o}}<8^{\circ}, \alpha<8^{\circ}, \delta<8^{\circ}$ & 35 & 64 \\
\hline & 2.3 & $V<3 \mathrm{~km} \mathrm{~s}^{-1}$, Dis $<6^{\circ}, \lambda_{\mathrm{o}}<8^{\circ}, \alpha<8^{\circ}, \delta<8^{\circ}$ & 207 & 389 \\
\hline & 2.4 & $V<0.5 \mathrm{~km} \mathrm{~s}^{-1}$, Dis $<2^{\circ}, \lambda_{\mathrm{o}}<8^{\circ}, \alpha<8^{\circ}, \delta<8^{\circ}$ & 13 & 20 \\
\hline & 2.5 & $V<1 \mathrm{~km} \mathrm{~s}^{-1}$, Dis $<2^{\circ}, \lambda_{\mathrm{o}}<8^{\circ}, \alpha<8^{\circ}, \delta<8^{\circ}$ & 23 & 42 \\
\hline & 2.6 & $V<3 \mathrm{~km} \mathrm{~s}^{-1}$, Dis $<2^{\circ}, \lambda_{\mathrm{o}}<8^{\circ}, \alpha<8^{\circ}, \delta<8^{\circ}$ & 128 & 240 \\
\hline \multirow{3}{*}{ Method 3} & 3.1 & $\mathrm{dv}=5 \%$, Dis $<2^{\circ}, \lambda_{\mathrm{o}}<8^{\circ}, \alpha<8^{\circ}, \delta<8^{\circ}$ & 36 & 55 \\
\hline & 3.2 & $\mathrm{dv}=10 \%$, Dis $<2^{\circ}, \lambda_{\mathrm{o}}<8^{\circ}, \alpha<8^{\circ}, \delta<8^{\circ}$ & 84 & 131 \\
\hline & 3.3 & $\mathrm{dv}=20 \%$, Dis $<2^{\circ}, \lambda_{\mathrm{o}}<8^{\circ}, \alpha<8^{\circ}, \delta<8^{\circ}$ & 251 & 367 \\
\hline
\end{tabular}

From Tables 1 and 2, we concluded that considering associations of asteroids with at least ten meteors is not enough to clearly identify new parent bodies. One can easily get a very large number of possible parent asteroids by slightly chasing the $N_{m}$ criterion. We decided to consider $N_{m} \geq 50$ to obtain a smaller number of possible associations (Table 2 ).

Using the first method (Table 2), and in the case of $D_{\text {sh }}<0.05$, the number of associations is small, knowing that normally we should obtain names of asteroids already known as parent bodies of some major meteor showers. Increasing the threshold value of the criterion to 0.1 or 0.2 did not solve the problem, and we obtained a very large number of possible associations with asteroids. The value of the chosen $D_{\text {sh }}$ criterion drastically changes the number of possible parent asteroids, thus preventing us from concluding with confidence regarding a potential parenthood.

On the other hand, using Methods 2 and 3 (Table 2) allowed us to obtain better constrain the potential parent bodies. Considering the preliminary results shown in Table 2 and the associations we obtained, we decided to consider the first case of the third method since the results are more robust $(\mathrm{dv}=5 \%$, Dis $<2^{\circ}, \lambda_{\mathrm{o}}<8^{\circ}, \alpha<8^{\circ}, \delta<8^{\circ}$ ).

Once this preliminary analysis is performed, the question remains: how can we test if the found associations are not simply resulting from a high number of meteoroid orbits? Such question was pertinently asked by Pauls \& Gladman (2005) regarding the Pribram and Neuschweinstein meteorites, and later used by Koten et al. (2014) to examine the possible associated meteor showers. The results were negative: the found possible associations between the two meteorites, as well as between each individual meteorite and meteors having similar orbital elements are simply the result of statistical fluctuations in a high set of orbits. As a consequence, we conduct a similar analysis for the 36 and 55 showers found with Method 3.1 (Table 2).

\subsection{Statistical test}

We use a slightly modified statistical test of Koten et al. (2014) to determine whether a given group of meteoroid orbits associated with a new parent body is a random coincidence or not. Let us assume that $N_{\text {tot }}$ is an amount of all recorded meteors within the corresponding interval of solar longitudes (excluding the shower meteors). $N_{\text {asoc }}$ is the number of meteors which were chosen as possible stream connected with a parent asteroid and $D_{\text {sh }}^{1, \ldots, N_{\text {asoc }}}$ are their D-criteria (Southworth \& Hawkins 1963). The statistical test itself is the following:

1. Artificial set of $N_{\text {tot }}$ orbits is created. The semimajor axes, eccentricities and inclinations have the same distribution as 
Table 3. Number of meteors associated with already known parent bodies.

\begin{tabular}{lrr}
\hline \hline Parent body & $N_{\mathrm{S}}$ & $N_{\mathrm{E}}$ \\
\hline $2011 X_{A 3}$ & 75 & 64 \\
$2009 \mathrm{WN}_{25}$ & 199 & - \\
$2004 T G_{10}$ & 70 & 93 \\
$2010 T U_{149}$ & 195 & 178 \\
$2015 D U_{180}$ & - & 55 \\
$(196256) 2003 E H_{1}$ & 2480 & 1976 \\
(3200) Phaethon $1983 \mathrm{~TB}$ & 14685 & 11778 \\
\hline
\end{tabular}

Notes. $N_{\mathrm{S}}$ : number of meteors in the SonotaCo database. $N_{\mathrm{E}}$ : number of meteors in the EDMOND Database.

small NEAs (absolute magnitude $H>24$, see Koten et al. 2014). Arguments of perihelion, longitudes of ascending node and mean anomalies are randomly chosen so that the simulated collisions with Earth occur in the same interval of solar longitudes as the observed meteor group.

2. For each artificial orbit the $D_{\mathrm{sh}}$-criterion (with respect to the possible parent body) is computed and these values are sorted so that

${\widetilde{D_{\mathrm{sh}}}}^{1}<{\widetilde{D_{\mathrm{sh}}}}^{2}<\cdots<{\widetilde{D_{\mathrm{sh}}}}^{N_{\mathrm{tot}}}$.

3. An occurence of a random subset which is "closer" to parent body than observed possible stream (and it is only random association) is tested by following the conditions:

${\widetilde{D_{\mathrm{sh}}}}^{1}<D_{\mathrm{sh}}^{1},{\widetilde{D_{\mathrm{sh}}}}^{2}<D_{\mathrm{sh}}^{2}, \ldots,{\widetilde{D_{\mathrm{sh}}}}^{N_{\text {asoc }}}<D_{\mathrm{sh}}^{N_{\text {asoc }}}$.

If all of these conditions are fulfilled, then a "false stream" is detected.

Steps $1-3$ are repeated $N=10000$ times. If $N_{\mathrm{f}}$ is a number of false streams, then probability of chance occurrence is

$P C O=N_{\mathrm{f}} / N$.

\section{Results}

As a first step to validate the method, we searched for already known associations between meteor showers and asteroids, for example, 3200 Phaethon and the Geminids. Table 3 presents the lists of such parent asteroids and the number of meteors found to be associated with each of them.

As a second step, we present in Table 4 two asteroids that have never been associated with meteor showers before, and for which the statistical tests is positive (the statistical significance of the association is high). Table 5 lists all unconfirmed cases, in order to illustrate the difficulty of the task, and the absolute necessity to perform statistical analysis in order to highlight potential parent bodies. Twenty six asteroids are potentially associated with meteors from both databases. Similarly, 28 asteroids are potentially associated with meteors either from SonotaCo or EDMOND Database only. This does not mean they were not associated with the other database at all, but the members of associations is less than 50 .

Examples of which meteor is potentially associated with which asteroid are provided in Tables A.1-A.5. By providing the community with such data, we hope to encourage future studies to complement and improve this work.
Table 4. Statistically not excluded new parent bodies and number of associated meteors in each database.

\begin{tabular}{lcccc}
\hline \hline Parent body & $\alpha$ & $\delta$ & $N_{\mathrm{S}}$ & $N_{\mathrm{E}}$ \\
\hline $2016 B N_{14}$ & 306.6169 & -5.3973 & 247 & 462 \\
$2015 T X_{24}$ & 49.3551 & 14.0942 & 179 & 278
\end{tabular}

Notes. $\alpha$ and $\delta$ are the location of the radiant (right ascension and declination, in deg. $N_{\mathrm{S}}$ : number of meteors in the SonotaCo database. $N_{\mathrm{E}}$ : number of meteors in the EDMOND Database.

We also computed the probability of chance occurrence (PCO) for each potential parent body associated with SonotaCo and EDMOND databases meteor orbits, using the method described in Sect. 2.3. The results are provided in Table 6. For the vast majority of potential parents, the statistical significance test prove the association to be the result of pure random process. Only for the first four asteroids (namely $2015 T X_{24}, 2016 B N_{14}$, $2015 Q T_{3}$ and $2009 H E_{21}$ ), the PCO is significantly lower than all other potential parents. However, even the PCO value for 2009 $H E_{21}$ is of $60 \%$ only, we decided not to consider it as a potential parent candidate. The $2015 Q T_{3}$ asteroid was only associated with 15 meteors from SonotaCo Database, which does not satisfy our condition in considering a potential parent body $\left(N_{m} \geq 50\right)$. However, considering the EDMOND data, we find 68 meteors possibly associated with this asteroid, which triggered the computation of PCO. Eventually, we found that $\mathrm{PCO}_{s}$ is very high, precisely because of the small number of meteors taken into account. Nevertheless, the $\mathrm{PCO}_{E}$ value $13 \%$ is quite low and statistically meaning that there are $87 \%$ of chances that the association is real. Overall, and as a consequence of this analysis, we decide that asteroids $2016 B N_{14}, 2015 T X_{24}, 2015 Q T_{3}$ are significant candidates to retain them, but ideally additional data would benefit from such a study to definitely confirm or infirm such conclusion. We should note that our work did not take into account the asteroids orbit uncertainties. In the case of poorly observed asteroid such as $2016 B N_{14}$ (observed for one day only) further observations leading to a new orbit determination might possibly change our results.

\section{Conclusion}

In this work, we searched in available databases (SonotaCo and EDMOND) for new meteor showers, with at least 50 members, identifying their parent bodies. Fifty four new asteroids were associated with meteor streams from both used databases. This is done by comparing their orbits basing on threshold values of the difference in geocentric velocity, distance to the radiant, right ascension, declination and solar longitude. We performed a statistical test to show whether or not the new meteor showers are only a chance occurrence. Our method ended up leading to much less confirmed associations than in absence of statistical test: from 26 asteroids potentially associated with at least 50 meteor orbits from SonotaCo and Edmond database, only three were not excluded. But at least they are identified using a reliable test. The two streams are associated with $2014 B N_{14}, 2015 T X_{24}$ and 2015 $Q T_{3}$ asteroids that were never linked to a meteor shower before.

Egal et al. (2017) and Vida et al. (2018) highlight the difficulty of computing an accurate meteoroid orbit from the observation of a meteor. In the light of such works, we understand today that our databases might contain lots of data in which accuracy are not as we previously thought they were. From these data, several new meteor showers are added regularly to the IAU. 
Table 5. Statistically excluded potential parent bodies.

\begin{tabular}{|c|c|c|c|c|}
\hline Parent body & $\alpha$ & $\delta$ & $N_{\mathrm{S}}$ & $N_{\mathrm{E}}$ \\
\hline $2011 U E_{256}$ & 54.35 & 10.01 & 594 & 793 \\
\hline $2003 W P_{21}$ & 53.14 & 15.40 & 451 & 568 \\
\hline $1999 V K_{12}$ & 56.52 & 9.28 & 303 & 373 \\
\hline $2007 R U_{17}$ & 36.79 & 7.82 & 262 & 381 \\
\hline $2016 U Z_{25}$ & 43.14 & 12.83 & 244 & 410 \\
\hline $2003 U V_{11}$ & 8.25 & 12.56 & 168 & 258 \\
\hline $2008 X M_{1}$ & 82.77 & 29.16 & 133 & 109 \\
\hline $2014 N K_{52}$ & 75.19 & 19.73 & 126 & 95 \\
\hline $2007 U L_{12}$ & 69.70 & 17.24 & 106 & 73 \\
\hline $2002 X M_{35}$ & 68.70 & 24.05 & 88 & 58 \\
\hline $2016 V K$ & 50.35 & 23.16 & 86 & 210 \\
\hline (251722) 1997 US2 & 70.82 & 17.90 & 82 & 70 \\
\hline $1997 V M_{4}$ & 63.84 & 10.33 & 82 & 112 \\
\hline $2014 U Q_{114}$ & 62.21 & 27.80 & 80 & 132 \\
\hline 2005 UR & 42.93 & 12.6 & 75 & 84 \\
\hline $2015 X L_{1}$ & 78.22 & 9.64 & 67 & - \\
\hline $2001 X X_{103}$ & 78.09 & 13.17 & 67 & - \\
\hline (312942) 1995 EK1 & 189.81 & 4.23 & 61 & 60 \\
\hline $2016 P Z_{39}$ & 94.05 & 19.63 & 61 & 57 \\
\hline $2014 Y Q_{34}$ & 104.10 & 25.89 & 59 & 53 \\
\hline $2014 W D_{7}$ & 55.42 & 21.69 & 59 & - \\
\hline $2006 S O_{198}$ & 27.99 & 4.36 & 58 & 159 \\
\hline $2010 \mathrm{XZ}$ & 73.76 & 7.41 & 55 & - \\
\hline $2009 H E_{21}$ & 226.65 & -12.44 & 53 & 79 \\
\hline (452302) $1995 \mathrm{YR} 1$ & 118.26 & 17.58 & 52 & - \\
\hline $2016 B V_{14}$ & 313.06 & -4.75 & 51 & 121 \\
\hline (4197) Morpheus 1982 TA & 26.17 & -0.79 & 50 & 111 \\
\hline $2013 \mathrm{BP}_{45}$ & 282.19 & 50.88 & 50 & 278 \\
\hline $2014 N F_{64}$ & 299.04 & -5.90 & - & 143 \\
\hline $2010 R V_{3}$ & 9.56 & -1.92 & - & 55 \\
\hline $2015 T D_{144}$ & 31.27 & 10.71 & - & 58 \\
\hline $2015 F P_{33}$ & 188.17 & 1.41 & - & 58 \\
\hline $2014 B D_{33}$ & 333.93 & 51.97 & - & 51 \\
\hline (139359) $2001 \mathrm{ME} 1$ & 280.70 & -18.86 & - & 55 \\
\hline $2014 H K_{197}$ & 205.72 & 0.01 & - & 50 \\
\hline $2016 D F_{1}$ & 316.67 & -14.21 & - & 107 \\
\hline $2013 R L_{43}$ & 13.16 & 10.10 & - & 91 \\
\hline 2016 TA & 24.17 & 5.22 & - & 85 \\
\hline (144861) 2004 LA12 & 291.92 & 59.50 & - & 99 \\
\hline $2011 G S_{60}$ & 335.54 & 1.56 & - & 74 \\
\hline $1999 V R_{6}$ & 40.31 & 24.44 & - & 70 \\
\hline $2015 Q O_{3}$ & 355.42 & 5.38 & - & 68 \\
\hline $2007 P R_{25}$ & 344.96 & -3.11 & - & 59 \\
\hline $2014 O X_{299}$ & 341.43 & -0.82 & - & 51 \\
\hline $2012 L L_{9}$ & 281.99 & 49.15 & - & 59 \\
\hline $2005 \mathrm{CM}_{7}$ & 316.75 & -8.41 & - & 56 \\
\hline $2015 P M_{307}$ & 344.92 & -1.46 & - & 58 \\
\hline (434677) 2006 BZ7 & 209.97 & 57.73 & - & 60 \\
\hline $2016 Q B_{11}$ & 342.15 & -4.32 & - & 50 \\
\hline $2015 Q T_{3}$ & 341.91 & -2.31 & - & 50 \\
\hline (297274) $1996 \mathrm{SK}$ & 21.94 & 11.43 & - & 54 \\
\hline
\end{tabular}

Notes. $\alpha$ and $\delta$ are the location of the radiant (right ascension and declination, in deg. $N_{\mathrm{S}}$ : number of meteors in the SonotaCo database. $N_{\mathrm{E}}$ : number of meteors in the EDMOND Database.
Table 6. Probability of chance occurrence (PCO) computed for each potential parent body associated with meteor orbits.

\begin{tabular}{|c|c|c|}
\hline Parent body & $P C O_{\mathrm{S}}(\%)$ & $P C O_{\mathrm{E}}(\%)$ \\
\hline $2015 T X_{24}$ & 24 & 10 \\
\hline $2015 Q T_{3}$ & 97 & 13 \\
\hline $2016 B N_{14}$ & 31 & 37 \\
\hline $2009 H E_{21}$ & 57 & 62 \\
\hline (139359) $2001 \mathrm{ME} 1$ & - & 77 \\
\hline (251722) $1997 U S_{2}$ & 96 & 82 \\
\hline $2016 B V_{14}$ & 87 & 83 \\
\hline $1999 V K_{12}$ & 90 & 84 \\
\hline $2003 U V_{11}$ & 91 & 84 \\
\hline $2014 Y Q_{34}$ & 96 & 84 \\
\hline $2015 F P_{33}$ & - & 84 \\
\hline $2007 R U_{17}$ & 79 & 86 \\
\hline $2008 X M_{1}$ & 92 & 88 \\
\hline $2003 W P_{21}$ & 93 & 91 \\
\hline 2016 TA & - & 92 \\
\hline $2006 S O_{198}$ & 96 & 92 \\
\hline $2013 R L_{43}$ & - & 93 \\
\hline $2011 U E_{256}$ & 96 & 94 \\
\hline $2010 R V_{3}$ & - & 95 \\
\hline $2016 U Z_{25}$ & 97 & 95 \\
\hline $2014 H K_{197}$ & - & 96 \\
\hline (297274) $1996 \mathrm{SK}$ & - & 96 \\
\hline $2016 Q B_{11}$ & - & 97 \\
\hline $2001 X X_{103}$ & 97 & 97 \\
\hline $2007 U L_{12}$ & 98 & 97 \\
\hline 2005 UR & 97 & 98 \\
\hline $2014 N K_{52}$ & 98 & 98 \\
\hline $2016 \mathrm{VK}$ & 98 & 98 \\
\hline (312942) $1995 E K_{1}$ & 98 & 98 \\
\hline $2015 T D_{144}$ & - & 98 \\
\hline $2015 Q O_{3}$ & - & 98 \\
\hline $2007 P R_{25}$ & - & 98 \\
\hline $2014 U Q_{114}$ & 97 & 99 \\
\hline $2002 X M_{35}$ & 99 & 99 \\
\hline $1997 \mathrm{VM}_{4}$ & 99 & 99 \\
\hline $2016 P Z_{39}$ & 99 & 99 \\
\hline (4197) Morpheus 1982 TA & 99 & 99 \\
\hline $2013 B_{45}$ & 99 & 99 \\
\hline $2014 N F_{64}$ & - & 99 \\
\hline $2014 B D_{33}$ & - & 99 \\
\hline $2016 D F_{1}$ & - & 99 \\
\hline (144861) $2004 L A_{12}$ & - & 99 \\
\hline $2011 G S_{60}$ & - & 99 \\
\hline $1999 V R_{6}$ & - & 99 \\
\hline $2014 O X_{299}$ & - & 99 \\
\hline $2012 L_{L}$ & - & 99 \\
\hline $2005 \mathrm{CM}_{7}$ & - & 99 \\
\hline $2014 W D_{7}$ & - & 99 \\
\hline $2015 P M_{307}$ & - & 99 \\
\hline (434677) $2006 \mathrm{BZ}_{7}$ & - & 99 \\
\hline $2015 X L_{1}$ & 98 & - \\
\hline $2010 \mathrm{XZ}$ & 99 & - \\
\hline (452302) $1995 Y R_{1}$ & 98 & - \\
\hline
\end{tabular}

Notes. The first three potential parent bodies have low values. $P C O_{\mathrm{S}}$ : PCO for meteors from the SonotaCo database; $P C O_{\mathrm{E}}$ : PCO for meteors from the EDMOND database. See text for further explanation. 
A meteor shower might sometimes be identified if only four to six meteors orbits are similar. Knowing the orbit real uncertainty (and inaccuracy...), and in absence of robust statistical test as the one shortly presented here, it is legitimate to question the veracity of the very existence of some meteor showers. It is worth mentioning that to revisit the list of meteor showers in the IAU database is a continuous ongoing work, and the reason for the very existence of several lists of meteor showers, including the "working list" and the "removed list". Even the statistical test performed in the present work might be improved in itself. We looked in the list of all meteor showers given by the IAU database, for streams associated with $2016 B N_{14}, 2015 T X_{24}$ and $2015 Q T_{3}$ asteroids. Neither of the three asteroids were associated with a stream from the list. This could be interpreted by the fact that these streams have never been observed before.

The present approach is actually pretty simple in its principle, and more experienced professional statisticians might come up with more robust ways to decide whether or not a possible parent body association is statistically significant or not. For example, Pauls \& Gladman (2005) revisited the possible association between the Neuschwanstein and Pribram meteorites and concluded by the negative.

In both SonotaCo and EDMOND databases, many orbits (all for SonotaCo) are obtained using the UFOOrbit software. Recently, SonotaCo (2016) provided the users with improved way to estimate the orbit accuracy. Ideally it would be worth revisiting all meteoroid orbit and to (re-)compute all of them using the latest methods (Vida et al. 2018). This would greatly help to revise, for example, the present work, but also will greatly help future works searching for meteor showers parent bodies.

Acknowledgements.We are thankful to L. Neslusan for providing us with the Neslusan et al. (1998) program.

\section{References}

Abedin, A., Wiegert, P., Pokorný, P., \& Brown, P. 2017, Icarus, 281, 417 Andreić, Ž., Šegon, D., Korlević, K., et al. 2013, WGN J. Int. Meteor Organ., 41, 103

Drummond, J. D. 1981, Icarus, 45, 545

Egal, A., Gural, P. S., Vaubaillon, J., Colas, F., \& Thuillot, W. 2017, Icarus, 294, 43

Hasegawa, I. 1990, PASJ, 42, 601

Jenniskens, P., \& Nénon, Q. 2016, Icarus, 266, 371

Jenniskens, P., Jopek, T. J., Rendtel, J., et al. 2009, WGN J. Int. Meteor Organ., 37,19

Jenniskens, P., Nénon, Q., Gural, P. S., et al. 2016, Icarus, 266, 384

Jopek, T. J. 1993, Icarus, 106, 603

Jopek, T. J., \& Bronikowska, M. 2017, Planet. Space Sci., 143, 43

Jopek, T. J., \& Kaňuchová, Z. 2017, Planet. Space Sci., 143, 3

Kornoš, L., Matlovič, P., Rudawska, R., et al. 2014, Meteoroids 2013, 225

Koten, P., Vaubaillon, J., Čapek, D., et al. 2014, Icarus, 239, 244

Lindblad, B. A. 1971a, Smithson. Contrib. Astrophys., 12, 14

Lindblad, B. A. 1971b, Smithson. Contrib. Astrophys., 12, 1

Micheli, M. 2013, Ph.D. Thesis, University of Hawai'i at Manoa, USA

Micheli, M., Tholen, D. J., \& Jenniskens, P. 2016, Icarus, 267, 64

Neslusan, L., Svoren, J., \& Porubcan, V. 1998, A\&A, 331, 411

Pauls, A., \& Gladman, B. 2005, Meteorit. Planet. Sci., 40, 1241

Porter, J. G. 1952, Comets and Meteor Streams (London: Chapman \& Hall)

Rudawska, R., \& Jenniskens, P. 2014, Meteoroids 2013, 217

Rudawska, R., Vaubaillon, J., \& Atreya, P. 2012, A\&A, 541, A2

Rudawska, R., Matlovic, P., Toth, J., Kornos, L., \& Hajdukova, M. 2015a, European Planetary Science Congress, 10, EPSC2015

Rudawska, R., Matlovič, P., Tóth, J., \& Kornoš, L. 2015b, Planet. Space Sci., 118, 38

Šegon, D., Andreić, Ž., Gural, P., et al. 2014, WGN J. Int. Meteor Organ., 42, 227 SonotaCo. 2009, WGN J. Int. Meteor Organ., 37, 55

SonotaCo. 2016, WGN J. Int. Meteor Organ., 44, 42

Southworth, R. B., \& Hawkins, G. S. 1963, Smithson. Contrib. Astrophys., 7, 261

Steel, D. I., \& Baggaley, W. J. 1985, MNRAS, 212, 817

Svoren, J., Neslusan, L., \& Porubcan, V. 1993, Contrib. Astron. Observ. Skalnate Pleso, 23, 23

Vaubaillon, J. 2017, Planet. Space Sci., 143, 78

Vida, D., Brown, P. G., \& Campbell-Brown, M. 2018, MNRAS, 479, 4307 


\section{Appendix A: Additional tables}

Table A.1. Orbital elements of the confirmed parent bodies.

\begin{tabular}{cccccccccc}
\hline \hline$\alpha_{\mathrm{pb}}$ & $\delta_{\mathrm{pb}}$ & $V_{g \mathrm{pb}}$ & $\lambda_{\mathrm{pb}}$ & $q_{\mathrm{pb}}$ & $e_{\mathrm{pb}}$ & $\omega_{\mathrm{pb}}$ & $i_{\mathrm{pb}}$ & $a_{\mathrm{pb}}$ & Desig \\
\hline 306.6169 & -5.3973 & 22.5479 & 127.5492 & 0.5814 & 0.7543 & 268.9198 & 10.1019 & 2.3664 & $(2016$ BN14) \\
49.3551 & 14.0942 & 31.2848 & 33.0070 & 0.2896 & 0.8724 & 127.0160 & 6.0442 & 2.2689 & (2015 TX24) \\
\hline
\end{tabular}

Notes. $\alpha_{\mathrm{pb}}, \delta_{\mathrm{pb}}, V_{g \mathrm{pb}}, q_{\mathrm{pb}}, e_{\mathrm{pb}}, a_{\mathrm{pb}}, \omega_{\mathrm{pb}}, \Omega_{\mathrm{pb}}, i_{\mathrm{pb}}$ and $\lambda_{\mathrm{pb}}$ refer, respectively, to right ascension $\left(^{\circ}\right)$ and declination $\left({ }^{\circ}\right)$ of the radiant, geocentric velocity $\left(\mathrm{km} \mathrm{s}^{-1}\right)$, perihelion distance $(\mathrm{au})$, eccentricity, semi-major axis $\left(^{\circ}\right)$, argument of perihelion $\left(^{\circ}\right)$, longitude of the ascending node $\left(^{\circ}\right)$, inclination $\left(^{\circ}\right)$ and solar longitude $\left(^{\circ}\right)$. "Desig" is the designation of the parent body.

Table A.2. Associations of meteors from SonotaCo Database with the $2003 W P_{21}$ asteroid.

\begin{tabular}{|c|c|c|c|c|c|c|c|c|c|c|c|c|}
\hline$\alpha$ & $\delta$ & $V_{g}$ & $\lambda$ & $q$ & $e$ & $\omega$ & $i$ & $a$ & Dis & $D_{\mathrm{sh}}$ & Desig & meteor_localtime \\
\hline 301.8032 & -11.1678 & 21.8474 & 121.0263 & 0.5777 & 0.7299 & 271.2687 & 6.5898 & 2.1390 & 1.4535 & 0.0863 & (2016 BN14) & 20080724_004307 \\
\hline & -10.2344 & & & & & & & & & & & 20080727_225158 \\
\hline 305.5989 & -8.8113 & 22.8116 & 125.7056 & 5759 & 7662 & 269.8716 & 7.9378 & 2.4628 & .0110 & 0419 & (2016 BN14) & 20080728_221536 \\
\hline 308.5972 & -9.32 & 23.3884 & 3.61 & 0.5074 & & 0.0117 & & & 1.9562 & 0.1277 & (2016 BN14) & 6_234851 \\
\hline 300.0231 & -11.58 & 21.6901 & 41 & 0.6294 & 0.7706 & 84 & & 2.7432 & 0.32 & & (201 & 01121 \\
\hline 08.0497 & -8.9481 & 22.8925 & 28.7472 & 5813 & 776 & 74 & & 2.6141 & & & (2016 BN14) & 5050 \\
\hline 306.3273 & -8.4300 & 22.2989 & 128.9342 & 6091 & 7751 & 65.1738 & 6654 & 2.7081 & 923 & 535 & (2016 BN14) & 13219 \\
\hline 308.0395 & -7.3520 & 22.6145 & 0.7534 & 0.6058 & 0.7852 & 35 & & 09 & & & $(2016 \mathrm{BI}$ & 230926 \\
\hline 312.9036 & -7.9640 & 22.51 & 134.5652 & 0.5938 & 0.7758 & 267.07 & 9250 & 2.6 & 0.0 & 0.0929 & (2016 BN14) & 24209 \\
\hline 302.3354 & -10.7849 & 23.4034 & 36 & 0.5653 & 0.7844 & 270.5 & & 16 & & 0.0 & (2016 & 5701 \\
\hline 304.8714 & -6.8 & & & & & & & & & & & 1504 \\
\hline 304.8480 & -9.18 & 23.03 & & & & & & & & & $(20$ & 23211 \\
\hline 305.3420 & -9.28 & 23.2914 & 96 & 0.5750 & 0.7873 & 269.1 & 7.7 & 2.70 & 1.26 & & $(201$ & 00522 \\
\hline 308.2440 & -9.15 & & & 0.5986 & & & & 2.4000 & & & (201 & 33730 \\
\hline 306.4201 & -8.18 & 2.8519 & 5846 & 0.6098 & 0.8008 & 4.1124 & & 0616 & & & (201 & 0814 \\
\hline 305.5402 & -7.8979 & 22.8785 & 129.5847 & 0.6196 & 0.8112 & 262.5076 & 8.2315 & 3.2810 & 95 & 0.09 & (2016 BN14) & 2_000815 \\
\hline 308.2933 & -8.2444 & 22.8551 & 129.5964 & 0.5879 & 0.7811 & 267.6736 & 7.76 & 2.6852 & 1.66 & & (2016 B & 02_002601 \\
\hline 306.9762 & -8.1993 & 22.2909 & 129.6281 & 0.6091 & 0.7753 & 265.1475 & & 2.7106 & 0.3 & 0.05 & (2016 BN14) & 02_011342 \\
\hline 307.4418 & -8.5479 & 22.1994 & & 0.6049 & 0.7683 & 265.9 & 7.3942 & 2.6113 & 0.8 & 0.0 & (2016 & 11432 \\
\hline 307.1376 & -10.4606 & 23.5177 & 129.6583 & 0.5978 & 0.8264 & 264.7393 & 6.3970 & 3.4432 & 0.5230 & 0.1025 & (2016 BN14) & 2_015939 \\
\hline 308.0323 & -8.7808 & 21.6655 & 129.6739 & 0.6042 & 0.7447 & 267.0128 & 7.0043 & 2.3670 & 1.4034 & & (2016 BN14) & 20120802_022253 \\
\hline 307.5287 & -8.8114 & 22.2418 & 129.6927 & 0.6045 & 0.7702 & 265.9410 & 7.2055 & 2.6308 & 0.9061 & 0.0592 & (2016 BN14) & 20120802_025108 \\
\hline 306.8643 & -9.7344 & 1.5444 & 129.7227 & 0.6202 & 0.7557 & 264.4636 & 6.4045 & 2.5384 & 0.2566 & 0.0814 & (2016 BN14) & 20120802_033632 \\
\hline 305.9524 & -9.3681 & 22.2413 & 126.5737 & 0.5879 & 0.7541 & 268.8032 & 7.2221 & 2.3911 & 0.6623 & 0.0527 & (2016 BN14) & 20160729_211752 \\
\hline 306.5570 & -9.5212 & 22.0857 & 126.5853 & 0.5825 & 0.7442 & 269.9464 & 7.0094 & 2.2775 & 0.0933 & 0.0550 & (2016 BN14) & 20160729_213521 \\
\hline 308.4269 & -8.4027 & 22.2346 & 130.2011 & 0.5995 & 0.7652 & 266.7912 & 7.3720 & 2.5533 & 1.7922 & 0.0530 & (2016 BN14) & 20170802_222208 \\
\hline & & & & & & & & & & & & 20170805_000819 \\
\hline 308.1434 & -7.6994 & 22.7353 & 132.1978 & 0.6197 & 0.8075 & 262.5938 & 7.8700 & 3.2196 & 1.5145 & 0.0808 & (2016 BN14) & 20170805_002600 \\
\hline
\end{tabular}

Notes. $\alpha, \delta, V_{g}, q, e, a, \omega, \Omega, i$ and $\lambda$ refer, respectively, to right ascension $\left(^{\circ}\right)$ and declination $\left({ }^{\circ}\right)$ of the radiant, geocentric velocity $\left(\mathrm{km} \mathrm{s}^{-1}\right)$, perihelion distance (au), eccentricity, semi-major axis $\left({ }^{\circ}\right)$, argument of perihelion $\left({ }^{\circ}\right)$, longitude of the ascending node $\left(^{\circ}\right)$, inclination $\left(^{\circ}\right)$ and solar longitude $\left(^{\circ}\right)$ of the meteors orbits. "Dis" is the distance to the radiant. We kept displaying the $D_{\text {sh }}$ criterion although we did not use it as a condition for associations. And over all, the criterion is less than 0.25 . For few associations with asteroids orbits, which have a large semi-major axis $a$, it exceeds this value up to 1.5. The larger the semi-major axis of the orbits the larger the value of $D_{\mathrm{sh}}$. For few associations with asteroids orbits, which have a large semi-major axis $a$, it exceeds this value up to 1.5. The larger the semi-major axis of the orbits the larger the value of $D_{\text {sh. }}$ The same quantities are represented in Tables A.3-A.5. 
A\&A 622, A84 (2019)

Table A.3. Associations examples of meteors from EDMOND database with the 2016 BN14 asteroid.

\begin{tabular}{|c|c|c|c|c|c|c|c|c|c|c|c|c|}
\hline$\alpha$ & $\delta$ & $V_{g}$ & $\lambda$ & $q$ & $e$ & $\omega$ & $i$ & $a$ & Dis & $D_{\text {sh }}$ & Desig & meteor_localtime \\
\hline 304.6471 & -9.6390 & 21.7765 & 126.0663 & 0.6022 & 0.7465 & 267.2454 & 7.0270 & 2.3759 & 1.9450 & 0.0713 & (2016 BN14) & 2008_7_28_22_19_9 \\
\hline 306.9226 & -8.6326 & 21.6780 & 126.0854 & 0.5762 & 0.7197 & 271.9232 & 7.5380 & 2.0555 & 0.3086 & 0.0604 & (2016 BN14) & 2008_7_28_22_47_38 \\
\hline 305.1463 & -9.3519 & 22.1343 & 126.1221 & 0.5933 & 0.7536 & 268.1283 & 7.3041 & 2.4075 & 1.4570 & 0581 & (2016 BN14) & 2008_7_28_23_43_1 \\
\hline 301.9407 & -12.2313 & 22.7363 & 119.7906 & 0.5525 & 0.7493 & 273.7175 & 6.1372 & 2.2040 & 1.5861 & 0.0862 & (2016 BN14) & 2010_7_22_20_47_28 \\
\hline 301.5560 & -12.0931 & 23.4388 & 119.9826 & 0.5520 & 0.7771 & 272.5310 & 6.4530 & 2.4771 & 1.2109 & 0.0920 & (2016 BN14) & 2010_7_23_1_37_20 \\
\hline 302.0199 & -11.3923 & 23.6481 & 120.8906 & 0.5548 & 0.7871 & 271.7480 & 6.9511 & 2.6053 & 1.6647 & 0.0875 & (2016 BN14) & _0_27_23 \\
\hline 301.9251 & -4.1936 & 23.0282 & 123.7269 & 0.5969 & 0.7729 & 266.8808 & 11.8764 & 2.6281 & 1.5844 & 0.0875 & (2016 BN14) & 3445 \\
\hline 305.3061 & -10.8479 & 22.8213 & 121.6723 & 0.5321 & 0.7384 & 276.9582 & 6.7932 & 2.0340 & 1.2984 & 0.0841 & (2016 BN14) & 2011_7_25_2_17_1 \\
\hline 304.6568 & -11.8925 & 22.1821 & 123.4884 & 0.5698 & 0.7419 & 271.7630 & 5.7138 & 2.2076 & 1.9288 & 0.0805 & (2016 BN14) & 2011_7_26_23_53_37 \\
\hline 304.9435 & -10.8656 & 22.8710 & 123.4947 & 0.5581 & 0.7592 & 272.5134 & 6.6570 & 2.3175 & 1.6533 & 0.0657 & (2016 BN14) & 2011_7_27_0_3_5 \\
\hline 311.2097 & -7.3692 & 23.0029 & 131.8742 & 0.5773 & 0.7780 & 269.1385 & 7.9630 & 2.6002 & 1.6767 & 0.0756 & (2016 BN14) & 2012_8_4_0_34_46 \\
\hline 302.2420 & -12.0989 & 22.9652 & 120.0277 & 0.5491 & 0.7564 & 273.8444 & 6.2592 & 2.2537 & 1.8782 & 0.0836 & (2016 BN14) & 2013_7_22_21_22_37 \\
\hline 301.3202 & -9.6764 & 22.7085 & 121.0348 & 0.5741 & 0.7586 & 270.4616 & 8.0102 & 2.3780 & 0.9798 & 0.0767 & (2016 BN14) & 2013_7_23_22_41_31 \\
\hline 301.2027 & -10.7145 & 21.6324 & 121.0999 & 0.5877 & 0.7273 & 270.0485 & 6.8867 & 2.1552 & 0.8645 & 0.0938 & (2016 BN14) & 2013_7_24_0_19_58 \\
\hline 302.1542 & -10.4230 & 21.9271 & 122.8951 & 0.5940 & 0.7454 & 268.4035 & 6.9699 & 2.3327 & 1.7977 & 0.0886 & (2016 BN14) & 2013_7_25_21_27_12 \\
\hline 301.3405 & -13.1056 & 22.6894 & 122.9449 & 0.5981 & 0.7890 & 266.1126 & 5.2735 & 2.8348 & 1.0007 & 0.1361 & (2016 BN14) & 2013_7_25_22_42_51 \\
\hline 306.5231 & -7.9279 & 21.8171 & 129.2869 & 0.6151 & 0.7580 & 265.0399 & 7.8102 & 2.5419 & 0.1031 & 0.0599 & (2016 BN14) & 2014_8_1_19_51_41 \\
\hline 307.0045 & -8.3326 & 22.1704 & 129.3866 & 0.6075 & 0.7680 & 265.6423 & 7.5971 & 2.6192 & 0.3881 & 0.0564 & (2016 BN14) & 2014_8_1_22_21_51 \\
\hline 306.9827 & -8.8554 & 22.1779 & 129.4041 & 0.6081 & 0.7710 & 265.4487 & 7.2268 & 2.6552 & 0.3679 & 0.0633 & (2016 BN14) & 2014_8_1_22_48_11 \\
\hline 313.7518 & -12.1171 & 22.3427 & 134.9534 & 0.5971 & 0.7808 & 266.4309 & 3.7380 & 2.7242 & 0.8488 & 0.1331 & (2016 BN14) & 2015_8_8_0_11_5 \\
\hline 299.7755 & -10.2410 & 22.9124 & 120.3936 & 0.5833 & 0.7756 & 268.5656 & 7.7976 & 2.5988 & 0.5591 & 0.1107 & (2016 BN14) & 2016_7_23_1_4_24 \\
\hline 312.3218 & 2.2022 & 23.4941 & 134.7662 & 0.5929 & 0.7735 & 267.2748 & 14.8805 & 2.6173 & 0.5927 & 0.1156 & (2016 BN14) & 2016_8_7__1_41_20 \\
\hline
\end{tabular}

Notes. Same as Table A.2.

Table A.4. Associations examples of meteors from SonotaCo database with the 2015 TX24 asteroid.

\begin{tabular}{|c|c|c|c|c|c|c|c|c|c|c|c|c|}
\hline$\alpha$ & $\delta$ & $V_{g}$ & $\lambda$ & $q$ & $e$ & $\omega$ & $i$ & $a$ & Dis & $D_{\text {sh }}$ & Desig & meteor_localtime \\
\hline 43.1818 & 11.0696 & 30.5240 & 28.5036 & 0.2884 & 0.8649 & 122.1032 & 7.2689 & 2.1351 & 0.1195 & 0.1442 & (2015 TX24) & 20081022_005605 \\
\hline 49.8472 & 12.8092 & 30.2422 & 34.3635 & 0.2803 & 0.8553 & 123.8683 & 7.4797 & 1.9374 & 0.4788 & 0.0418 & (2015 TX24) & 20081027_220440 \\
\hline 49.7198 & 17.0200 & 32.3685 & 34.3753 & 0.2420 & 0.8933 & 127.1055 & 1.9753 & 2.2672 & 0.3548 & 0.0908 & (2015 TX24) & 20081027_222253 \\
\hline 49.8208 & 13.9842 & 30.3591 & 34.5622 & 0.2758 & 0.8583 & 124.3409 & 5.9421 & 1.9460 & 0.4515 & 0.0263 & (2015 TX24) & 20081028_025123 \\
\hline 48.0741 & 13.8526 & 30.4594 & 35.2887 & 0.3049 & 0.8703 & 119.3035 & 5.2224 & 2.3507 & 1.2372 & 0.0855 & (2015 TX24) & 20091029_021245 \\
\hline 50.5474 & 13.3462 & 30.4179 & 35.3834 & 0.2806 & 0.8597 & 123.5157 & 7.0217 & 1.9995 & 1.1539 & 0.0292 & (2015 TX24) & 20091029_043020 \\
\hline 53.8185 & 8.0276 & 31.6086 & 40.8277 & 0.3365 & 0.8981 & 113.3620 & 14.4765 & 3.3021 & 1.7749 & 0.1827 & (2015 TX24) & 20101103_212640 \\
\hline 55.9759 & 15.9776 & 31.2782 & 40.9638 & 0.2682 & 0.8753 & 124.2707 & 5.4260 & 2.1516 & 0.3276 & 0.0836 & (2015 TX24) & 20101104_004155 \\
\hline 42.0675 & 9.7891 & 29.8091 & 29.7871 & 0.3324 & 0.8633 & 115.9308 & 7.7530 & 2.4309 & 0.9833 & 0.2223 & (2015 TX24) & 20111024_021850 \\
\hline 47.9268 & 11.2606 & 30.1309 & 33.6409 & 0.3028 & 0.8579 & 120.3826 & 8.5104 & 2.1317 & 1.3871 & 0.1022 & (2015 TX24) & 20111027_230421 \\
\hline 49.0795 & 14.8858 & 30.1149 & 33.6596 & 0.2710 & 0.8534 & 125.4240 & 4.4537 & 1.8481 & 0.2671 & 0.0410 & (2015 TX24) & 20111027_233035 \\
\hline 50.9554 & 13.5110 & 30.6485 & 37.3398 & 0.2978 & 0.8704 & 120.2903 & 6.7619 & 2.2975 & 1.5417 & 0.0407 & (2015 TX24) & 20131031_041839 \\
\hline 44.0599 & 13.0214 & 32.7855 & 30.0719 & 0.2665 & 0.9094 & 122.4934 & 5.5288 & 2.9406 & 0.9583 & 0.1240 & (2015 TX24) & 20141024_033122 \\
\hline 48.5841 & 15.0229 & 32.1075 & 31.9911 & 0.2359 & 0.8859 & 128.5865 & 4.6334 & 2.0681 & 0.7452 & 0.0612 & (2015 TX24) & 20141026_014302 \\
\hline 47.6989 & 14.9591 & 31.3634 & 32.0261 & 0.2536 & 0.8756 & 126.5754 & 4.1252 & 2.0379 & 1.5879 & 0.0538 & (2015 TX24) & 20141026_023259 \\
\hline 50.4043 & 14.2206 & 30.3051 & 37.7589 & 0.3094 & 0.8675 & 118.8034 & 5.4681 & 2.3351 & 1.0142 & 0.0582 & (2015 TX24) & 20151101_023821 \\
\hline 54.1877 & 14.0211 & 30.9422 & 40.5586 & 0.2963 & 0.8755 & 120.1528 & 7.1993 & 2.3803 & 1.3981 & 0.0280 & (2015 TX24) & 20151103_214740 \\
\hline 54.0047 & 14.7095 & 30.3156 & 40.6506 & 0.3022 & 0.8638 & 120.0167 & 6.0458 & 2.2196 & 1.5678 & 0.0226 & (2015 TX24) & 20151103_235948 \\
\hline 54.5832 & 17.9022 & 31.5241 & 37.4374 & 0.2303 & 0.8741 & 130.2885 & 2.4221 & 1.8293 & 1.0122 & 0.1458 & (2015 TX24) & 20161031_005644 \\
\hline 49.5322 & 13.5175 & 30.7711 & 37.4697 & 0.3151 & 0.8809 & 117.2354 & 6.1003 & 2.6452 & 0.1723 & 0.0864 & (2015 TX24) & 20161031_014823 \\
\hline 55.6745 & 8.7791 & 30.7262 & 30.1024 & 0.1939 & 0.8489 & 138.8985 & 18.4465 & 1.2830 & 0.0993 & 0.2735 & (2015 TX24) & 20171023_223950 \\
\hline 48.5366 & 11.3304 & 32.7920 & 32.2095 & 0.2527 & 0.8994 & 125.1014 & 10.1918 & 2.5131 & 0.7987 & 0.0953 & (2015 TX24) & 20171026_012337 \\
\hline
\end{tabular}

Notes. Same as Table A.2. 
M. Guennoun et al.: SonotaCo and EDMOND meteoroid orbit databases

Table A.5. Associations examples of meteors from EDMOND database with the 2015 TX24 asteroid.

\begin{tabular}{|c|c|c|c|c|c|c|c|c|c|c|c|c|}
\hline$\alpha$ & $\delta$ & $V_{g}$ & $\lambda$ & $q$ & $e$ & $\omega$ & $i$ & $a$ & Dis & $D_{\mathrm{sh}}$ & Desig & _localtime \\
\hline 8.2506 & 14.0420 & 30.9687 & 39.8319 & 0.3576 & 0.9124 & 109.9187 & 4.6711 & 4.0804 & 1.0677 & 0.1805 & (2015 TX24) & 2008_11_2_0_20_18 \\
\hline & & & & & & & & & & & & \\
\hline & & & & & & & & & & & & \\
\hline 47.7460 & 3587 & & & 0.30 & 3522 & 119.8 & & & & & 4) & 8_21 \\
\hline & & & & & & & & & & & & $\_25$ \\
\hline 374 & 856 & & & 0.28 & & & & & 0.0 & & & _32 \\
\hline & & & & & & 1 & & & & & 01 & \\
\hline 847 & 019 & & & 8 & 8866 & 111.8 & 78 & & 1.5 & & $(201$ & 29 \\
\hline 50.5975 & 13.5060 & & 36.2 & & & & & & & & & 2010 \\
\hline & 2240 & & .0083 & 0.28 & & & & & & & & 201 \\
\hline 41.9312 & 11.8952 & 30.74 & & & & & & & & & $(20$ & \\
\hline .9726 & 654 & 31.1 & & 6 & & & & & & & & 44 \\
\hline 42.6148 & 12.6891 & 32.0239 & & & & & & & & & & 2012 \\
\hline 50.1872 & 15.5907 & 29.8819 & & 0.2668 & & 126.44 & & & 0.8 & & $(2015)$ & 2013 \\
\hline 5799 & & & & & & & & & & & & t_15 \\
\hline 903 & 9092 & & & 2276 & 336 & 130.1 & & & & & (2015 & 2014 \\
\hline 42.9334 & 15.4598 & 30.9782 & 26.2692 & 0.2373 & 0.8668 & & & 1.7821 & & & & 9_22_48_55 \\
\hline 50.1856 & 16.1529 & 31.4041 & 34.8809 & 0.2556 & 0.8768 & 126.1403 & 3.3129 & 2.0750 & 0.8008 & 0.0607 & (2015 TX24) & 3_20_27_38 \\
\hline & 13.0255 & & & & & & & 73 & & & & _27_39 \\
\hline & & & & & & & & & & & & 2015_10_30_2_25_42.0 \\
\hline 47.5397 & 12.5696 & 30.7491 & & & & & & & & & & 2016_10_28_22_49_46 \\
\hline 50.1987 & 7.1204 & 30.2040 & 35.7426 & 0.3315 & 0.8591 & 116.2207 & 14.0720 & 2.3531 & 0.8366 & 0.1911 & (2015 TX24) & 2016_10_28_23_22_48 \\
\hline
\end{tabular}

Notes. Same as Table A.2. 